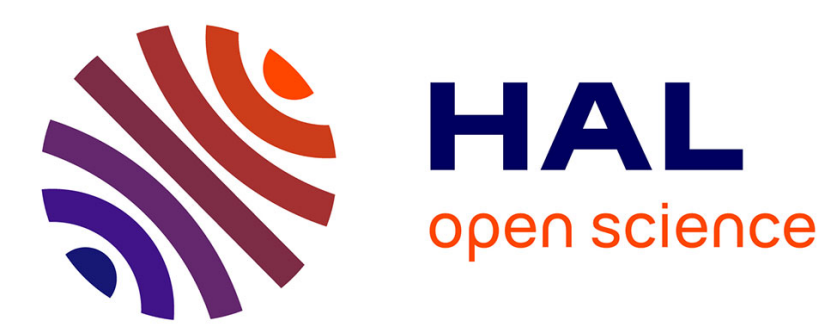

\title{
Hybrid fluid-quantum coupling for the simulation of the transport of partially quantized particles in a DG-MOSFET
}

\author{
Clément Jourdana, Nicolas Vauchelet
}

\section{- To cite this version:}

Clément Jourdana, Nicolas Vauchelet. Hybrid fluid-quantum coupling for the simulation of the transport of partially quantized particles in a DG-MOSFET. Mathematics of Quantum Technologies, 2015, 4 (1), pp.1-17. 10.1515/nsmmt-2015-0001 . hal-01159939

\section{HAL Id: hal-01159939 \\ https://hal.science/hal-01159939}

Submitted on 7 Jun 2015

HAL is a multi-disciplinary open access archive for the deposit and dissemination of scientific research documents, whether they are published or not. The documents may come from teaching and research institutions in France or abroad, or from public or private research centers.
L'archive ouverte pluridisciplinaire HAL, est destinée au dépôt et à la diffusion de documents scientifiques de niveau recherche, publiés ou non, émanant des établissements d'enseignement et de recherche français ou étrangers, des laboratoires publics ou privés. 


\title{
Hybrid fluid-quantum coupling for the simulation of the transport of partially quantized particles in a DG-MOSFET
}

\author{
C. Jourdana*, N. Vauchelet ${ }^{\dagger \ddagger \delta}$
}

June 3,2015

\begin{abstract}
This paper is devoted to numerical simulations of electronic transport in nanoscale semiconductor devices for which charged carriers are extremely confined in one direction. In such devices, like DG-MOSFETs, the subband decomposition method is used to reduce the dimensionality of the problem. In the transversal direction electrons are confined and described by a statistical mixture of eigenstates of the Schrödinger operator. In the longitudinal direction, the device is decomposed into a quantum zone (where quantum effects are expected to be large) and a classical zone (where they are negligible). In the largely doped source and drain regions of a DG-MOSFET, the transport is expected to be highly collisional; then a classical transport equation in diffusive regime coupled with the subband decomposition method is used for the modeling, as proposed in N. Ben Abdallah et al. (2006, Proc. Edind. Math. Soc. [7]). In the quantum region, the purely ballistic model presented in Polizzi et al. (2005, J. Comp. Phys. 25]) is used. This work is devoted to the hybrid coupling between these two regions through connection conditions at the interfaces. These conditions have been obtained in order to verify the continuity of the current. A numerical simulation for a DG-MOSFET, with comparison with the classical and quantum model, is provided to illustrate our approach.
\end{abstract}

Keywords: Schrödinger equation; subband decomposition; drift-diffusion system; semiconductors; interface conditions; mixed finite elements.

2010 AMS subject classifications: 65M60, 65Z05, 82D37, 82D80, 35J10, 76P05

\section{Introduction}

The constant downscaling at nanometer scales of electronic components allows to build devices more functionals. In order to fit the requirements of the International Technology Roadmap for the Semiconductor Industry [15], new technology has been developed such as the multi-gate devices. The use of Double Gate Metal Oxide Semiconductor Field Effect Transistors (DG-MOSFETs) seems to be mandatory in the near future since such device allows an outstanding control on the short channel effects arising in bulk MOSFET [12]. In this task, modeling and numerical simulation

\footnotetext{
${ }^{*}$ Université Grenoble Alpes, Laboratoire Jean Kuntzmann, F-38000 Grenoble, France. Email: clement. jourdana@imag.fr

${ }^{\dagger}$ Sorbonne Universités, UPMC Univ Paris 06, UMR 7598, Laboratoire Jacques-Louis Lions, F-75005, Paris, France, Email: nicolas.vauchelet@upmc.fr

${ }^{\ddagger}$ CNRS, UMR 7598, Laboratoire Jacques-Louis Lions, F-75005, Paris, France

${ }^{\S}$ INRIA-Paris-Rocquencourt, EPC MAMBA, Domaine de Voluceau, BP105, 78153 Le Chesnay Cedex, France
} 
play an important role to predict the behavior of such devices whose electron transport is strongly driven by quantum effects [2, 13, 16]. In nanoscale DG-MOSFETs electrons might be extremely confined in one direction (denoted $z$ and referred to as the confining direction) which implies a partial quantization of the energy. The subband decomposition method [31, 25] allows to reduce the dimensionality of the problem thanks to a splitting of the transport and confining direction. In the transversal direction, denoted $z$, electrons are confined in a small dimension and are described by a statistical mixture of eigenstates of the Schrödinger operator. In the transport directions $x$, the particles motion can be of quantum or classical nature.

In this work we focus on the description of the transport of partially confined particles in a Double-Gate MOSFET. The electron gas is supposed to be confined in the transversal direction $z$. The confinement induces a discretization of the energy continuum and thus the creation of energy-subbands in the transversal direction. In fact, a large potential barrier at these oxide-silicon interfaces induces a strong confinement in the $z$ direction. Therefore, the subband decomposition method is relevant for this electronic component. The electron motion is allowed in the remaining two directions. Assuming translational invariance, the simulation domain is reduced to a $2 \mathrm{D}$ domain $(x, z) \in[0, L] \times[0, \ell]$. A schematic representation of the regions for such a device is given in Figure 1. The electron transport is expected to operate in a quantum ballistic regime in the active region outside of the electron reservoirs. The source and drain zones are largely doped and collisions are then predominant in the transport. Therefore quantum phenomena are very localized in the channel between the source and drain regions.

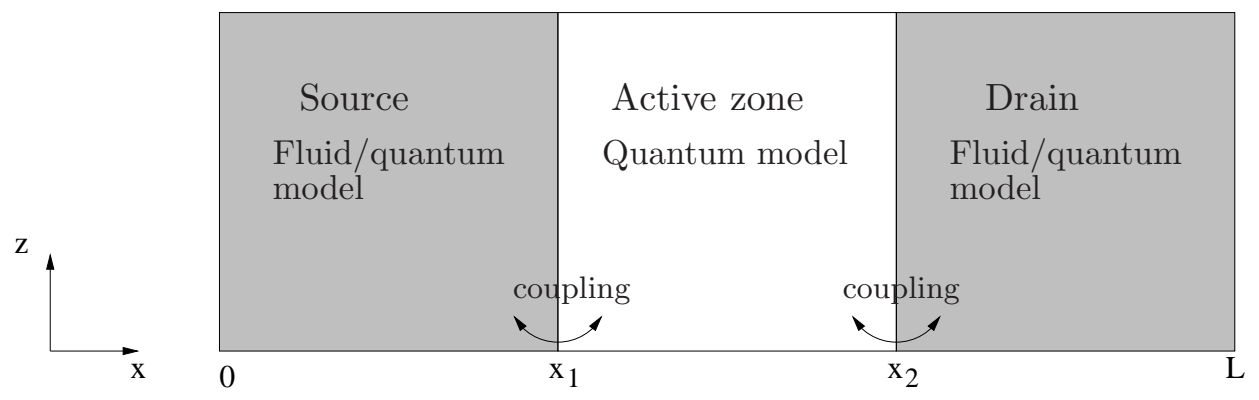

Figure 1: Schematic representation of the regions of the device. The coupling between the classical and the quantum regions occurs for $x=x_{1}$ and $x=x_{2}$.

To simulate the quantum ballistic transport, the subband decomposition method allows for a reduction of the numerical cost. In fact, the resolution of the Schrödinger equation in the whole domain is replaced by 1D eigenvalue problems in the confined (or transversal) direction $z$ and a system of coupled Schrödinger equations in the transport (or longitudinal) direction $x$. Then numerical simulations of the ballistic transport of confined particles in nanotransistors using the subband decomposition method are provided in [25]. In this latter article, the Schrödinger-Poisson system in the longitudinal direction is solved self-consistently via standard variational formulations in the whole domain. In 8, the authors have proposed to combine the subband decomposition method with the WKB method to reduce the cost of simulations. With their approach, the resolution of the Schrödinger equation in the longitudinal direction $x$ is accelerated through the use of WKB techniques. The WKB approximation has been used in this manner for the 1D simulation of a resonant tunneling diode in [9].

Such fully quantum model is convenient to simulate the ballistic transport. However, we may expect that in some region of the device of interest the transport is strongly affected by collisions. In fact, it is well-known that transport of charged carriers in semiconductors is highly collisional 
(see e.g. [19]). Then fluid-quantum coupled models where the coupling occurs in the momentum variable through the subband decomposition method have been investigated by several authors. In [7, 28, a model for the diffusive transport of partially quantized particles is proposed and analyzed. The transport in the $x$ direction is modeled by the drift-diffusion system for semi-conductors [21] coupled to the stationary Schrödinger-Poisson system in the $z$ direction. Using this model, numerical simulations of the diffusive transport of electrons confined in a DG-MOSFET have been obtained in 23. It has been stated in 29 that this drift-diffusion-Schrödinger-Poisson system is obtained by a diffusive limit of a Boltzmann-Schrödinger-Poisson system, where the transport in the $x$ direction is modeled by the Boltzmann transport equation in diffusive scaling. Numerical simulations of this kinetic-quantum model are provided in [30, 5]. At an intermediate level of description, an EnergyTransport model coupled with the subband decomposition method has been proposed and simulated in [6, 24].

The aim of this paper is to propose a hybrid coupling between quantum ballistic model in the channel and diffusive-quantum model in the highly collisional regions of the source and the drain. The coupling between the models is obtained through boundary conditions at the interface $x_{1}$ and $x_{2}$ between these regions (see Figure 1]). In his seminal work [3], N. Ben Abdallah proposes a coupled kinetic-quantum model for one-dimensional resonant tunneling diode. In his work, the Schrödinger equation is used in the quantum zone whereas, in the classical regions, a Boltzmann equation is used to describe the collisional transport of electrons in the rest of the domain. At the classical-quantum interface, boundary conditions for the Boltzmann equation depending on the quantum reflection and transmission coefficients have been defined. A numerical discretization of this approach has been later proposed in [10]. Aiming at diminishing computational cost, the diffusive limit of the Boltzmann equation leading to the drift-diffusion (DD) model has been considered in [14. In their paper, the authors have derived the interface boundary conditions by passing to the limit in the reflectiontransmission conditions obtained in [3] and by considering boundary layer corrections leading to the resolution to a Milne problem. The resolution of the Milne problem is quite involved and its numerical cost can be important. Then, another approach has been proposed in [1], where the authors derived boundary conditions by imposing the continuity of the current at the interfaces. It has been shown in particular that this strategy is a good approximation of the previous one. Moreover, since this approach does not involve the resolution of a Milne problem, its formulation is simpler and its numerical cost is reduced. Similar observation has been done for the Energy-Transport model in [18. We propose in this paper to follow the latter strategy and to extend the hybrid coupling to the subband decomposition method. Then by imposing continuity of the current at the interfaces, boundary conditions are derived. It allows for the numerical simulation of the transport of partially confined electron gas in a nanotransistor DG-MOSFET.

The outline of the paper is as follows. In Section 2, we describe the modeling and the description of the open boundary conditions at the interface. We first recall the subband decomposition approach in $\$ 2.1$ which describes the confinement in the transversal direction. Then we describe the models used in each region separately. In $₫ 2.2$, the quantum model describing the ballistic transport in the channel is recalled. Subsection 2.3 is devoted to the description of the fluid model used in the classical region. The interface conditions allowing to connect these two regions are obtained in $\$ 2.4$ using the continuity of the current. Finally, Section 3 is devoted to numerical results of the resulting model. 


\section{Modeling and boundary conditions}

\subsection{Subband decomposition method}

In the subband decomposition method, the system is viewed as a statistical mixture of eigenstates of the Schrödinger equation (see [25]). The strong confinement in the transversal direction $z \in(0, \ell)$ implies a quantization of the energy in level denoted $\epsilon_{k}$. From a mathematical viewpoint, these energy levels, called subbands, are eigenvalues of the 1D stationary Schrödinger operator whose eigenvectors denoted $\left(\chi_{k}\right)_{k \geq 1}$ form an orthonormal basis of $L^{2}(0, \ell)$ :

$$
\left\{\begin{array}{l}
-\frac{\hbar^{2}}{2 m^{*}} \frac{d^{2}}{d z^{2}} \chi_{k}+\left(U+U_{c}\right) \chi_{k}=\epsilon_{k} \chi_{k}, \\
\chi_{k}(x, 0)=\chi_{k}(x, \ell)=0, \quad\left\langle\chi_{k}(x, .) \mid \chi_{k^{\prime}}(x, .)\right\rangle=\delta_{k k^{\prime}} .
\end{array}\right.
$$

In this equation and in the sequel, we will always use the following notations: $\delta_{k k^{\prime}}$ is the Kronecker delta (i.e. $\delta_{k k^{\prime}}=1$ if $k=k^{\prime}, \delta_{k k^{\prime}}=0$ if $k \neq k^{\prime}$ ),

$$
\left\langle\chi_{k}(x, .) \mid \chi_{k^{\prime}}(x, .)\right\rangle=\int_{0}^{\ell} \chi_{k}(x, z) \chi_{k^{\prime}}(x, z) d z .
$$

In equation (2.1) $\hbar$ denotes the reduced Planck constant, $m^{*}$ the effective mass and $U_{c}$ is a given potential barrier at the interface between the oxide and the silicon.

The electrostatic potential energy $U$ is defined by $U=-e V$, where $e$ is the elementary charge and $V$ denotes the self-consistent electrostatic potential solution of the Poisson equation

$$
-\operatorname{div}{ }_{x, z}\left(\varepsilon_{r}(x, z) \nabla_{x, z} V\right)=\frac{e}{\varepsilon_{0}}\left(N_{D}-N\right) .
$$

Here $\varepsilon_{r}(x, z)$ is the relative dielectric permittivity, $\varepsilon_{0}$ the constant permittivity of vacuum and $N_{D}(x, z)$ is the given doping density. The density of electrons is denoted $N(x, z)$. Its expression depends on the transport direction and is detailed in the following. This Poisson equation should be complemented with boundary conditions at the frontier of the simulation domain $[0, L] \times[0, \ell]$. We will divide the boundary into $\Gamma_{D}$ where ohmic contacts impose a fixed potential (such as the drain, source and gates contacts) and $\Gamma_{N}$ where there insulating conditions are considered. Then we impose Dirichlet boundary conditions on $\Gamma_{D}$ and homogeneous Neumann boundary conditions on $\Gamma_{N}$ :

$$
V(x, z)=V_{b}, \quad \text { for }(x, z) \in \Gamma_{D} ; \quad \partial_{n} V(x, z)=0, \quad \text { for }(x, z) \in \Gamma_{N} ;
$$

where we denote by $V_{b}$ a given applied bias at the Gate or Drain or Source contacts.

\subsection{The quantum region}

This Section is devoted to the modeling of the transport in the quantum region. We recall, see Figure 1 that the quantum region is sandwiched between two classical regions and corresponds to $x \in \Sigma=\left(x_{1}, x_{2}\right)$. The subband decomposition method for the ballistic transport has been introduced in 25]. We recall here the resulting system of equations, which consists in two stationary Schrödinger equations in the transport direction with open transmitting boundary conditions, for each energy level computed thanks to (2.1) in the transversal direction.

To simplify the presentation, we first assume that the electrostatic potential $V$ is known in the whole device. Then thanks to a diagonalization of the stationary Schrödinger operator (2.1), the set 
$\left(\epsilon_{k}, \chi_{k}\right)_{k \geq 1}$ of eigenstates is known. We define the potential $\widetilde{V}$ by

$$
\tilde{V}(x)= \begin{cases}V_{1}:=V\left(x_{1}\right) & x \leq x_{1}, \\ V(x) & x_{1} \leq x \leq x_{2}, \\ V_{2}:=V\left(x_{2}\right) & x \geq x_{2} .\end{cases}
$$

This is a continuous extension of $V$ by a constant on $\Omega=(0, L) \backslash \Sigma$. We define the energy $E_{k, 1}$ and respectively $E_{k, 2}$ at the interfaces $x=x_{1}$ and respectively $x=x_{2}$ by

$$
E_{k, 1}(p):=\frac{p^{2}}{2 m^{*}}+\epsilon_{k}\left(x_{1}\right) \quad ; \quad E_{k, 2}(p):=\frac{p^{2}}{2 m^{*}}+\epsilon_{k}\left(x_{2}\right)
$$

where $p$ denotes the moment variable and $\epsilon_{k}$ the potential energy of the $k$ th subband, defined in (2.1). The wave vector is given by

$$
p_{j}^{\ell}\left(E_{k, i}(p)\right):=\sqrt{2 m^{*}\left|E_{k, i}(p)-\epsilon_{\ell}\left(x_{j}\right)\right|}=\sqrt{\mid p^{2}+2 m^{*}\left(\epsilon_{k}\left(x_{i}\right)-\epsilon_{\ell}\left(x_{j}\right) \mid\right.},
$$

where $i$ and $j$ take the values 1 or 2 .

The stationary Schrödinger operator is defined by

$$
H=-\frac{\hbar^{2}}{2 m^{*}}\left(\frac{\partial^{2}}{\partial x^{2}}+\frac{\partial^{2}}{\partial z^{2}}\right)
$$

Let $\psi_{k}^{+}$be the wave function entering the quantum zone in $x_{1}$ on the $k$ th subband and having the energy $E_{k, 1}(p)$ (2.4), and respectively $\psi_{k}^{-}$is the wave function entering in $x_{2}$ on the $k$ th subband with energy $E_{k, 2}(p)$. For each momentum $p$, the wave functions $\psi_{k}^{ \pm}$are then solutions of the Schrödinger equation with open boundary conditions. This system reads (see [20, 4, 25]) :

$$
\left\{\begin{aligned}
& H \psi_{k}^{+}+\widetilde{V} \psi_{k}^{+}=E_{k, 1}(p) \psi_{k}^{+}, \quad \text { for }(x, z) \in\left[x_{1}, x_{2}\right] \times(0, \ell) \\
& \psi_{k}^{+}(x, 0)=\psi_{k}^{+}(x, \ell)=0, \\
& \hbar \frac{\partial \psi_{k}^{+}}{\partial x}\left(x_{1}, z\right)=2 \mathbf{i} p \chi_{k}\left(x_{1}, z\right)-\sum_{j=1}^{M^{1}\left(E_{k, 1}(p)\right)} \mathbf{i} p_{1}^{j}\left(E_{k, 1}(p)\right)\left\langle\psi_{k}^{+}\left(x_{1}, \cdot\right) \mid \chi_{j}\left(x_{1}, \cdot\right)\right\rangle \chi_{j}\left(x_{1}, z\right) \\
& \quad+\sum_{j=M^{1}\left(E_{k, 1}(p)\right)+1}^{\infty} p_{1}^{j}\left(E_{k, 1}(p)\right)\left\langle\psi_{k}^{+}\left(x_{1}, \cdot\right) \mid \chi_{j}\left(x_{1}, \cdot\right)\right\rangle \chi_{j}\left(x_{1}, z\right) \\
& \hbar \frac{\partial \psi_{k}^{+}}{\partial x}\left(x_{2}, z\right)=\sum_{j=1}^{M^{2}\left(E_{k, 1}(p)\right)} \mathbf{i} p_{2}^{j}\left(E_{k, 1}(p)\right)\left\langle\psi_{k}^{+}\left(x_{2}, \cdot\right) \mid \chi_{j}\left(x_{2}, \cdot\right)\right\rangle \chi_{j}\left(x_{2}, z\right) \\
&-\sum_{j=M^{2}\left(E_{k, 1}(p)\right)+1}^{\infty} p_{2}^{j}\left(E_{k, 1}(p)\right)\left\langle\psi_{k}^{+}\left(x_{2}, \cdot\right) \mid \chi_{j}\left(x_{2}, \cdot\right)\right\rangle \chi_{j}\left(x_{2}, z\right)
\end{aligned}\right.
$$

where

$$
M^{j}\left(E_{k, i}(p)\right)=\sup \left\{\ell \in \mathbb{N}^{*} \text { such that } \epsilon_{\ell}\left(x_{j}\right) \leq E_{k, i}(p)\right\}
$$


And

$$
\left\{\begin{array}{l}
H \psi_{k}^{-}+\tilde{V} \psi_{k}^{-}=E_{k, 2}(p) \psi_{k}^{-}, \quad \text { for }(x, z) \in\left[x_{1}, x_{2}\right] \times(0, \ell) . \\
\psi_{k}^{-}(x, 0)=\psi_{k}^{-}(x, \ell)=0, \\
\hbar \frac{\partial \psi_{k}^{-}}{\partial x}\left(x_{1}, z\right)=-\sum_{j=1}^{M^{1}\left(E_{k, 2}(p)\right)} \mathbf{i} p_{1}^{j}\left(E_{k, 2}(p)\right)\left\langle\psi_{k}^{-}\left(x_{1}, \cdot\right) \mid \chi_{j}\left(x_{1}, \cdot\right)\right\rangle \chi_{j}\left(x_{1}, z\right) \\
\quad+\sum_{j=M^{1}\left(E_{k, 2}(p)\right)+1}^{\infty} p_{1}^{j}\left(E_{k, 2}(p)\right)\left\langle\psi_{k}^{-}\left(x_{1}, \cdot\right) \mid \chi_{j}\left(x_{1}, \cdot\right)\right\rangle \chi_{j}\left(x_{1}, z\right), \\
\hbar \frac{\partial \psi_{k}^{-}}{\partial x}\left(x_{2}, z\right)=-2 \mathbf{i} p \chi_{k}\left(x_{2}, z\right)-\sum_{j=1}^{\left.M_{k, 2}(p)\right)} \mathbf{i} p_{2}^{j}\left(E_{k, 2}(p)\right)\left\langle\psi_{k}^{-}\left(x_{2}, \cdot\right) \mid \chi_{j}\left(x_{2}, \cdot\right)\right\rangle \chi_{j}\left(x_{2}, z\right) \\
\quad \sum_{j=M^{2}\left(E_{k, 2}(p)\right)+1}^{\infty} p_{2}^{j}\left(E_{k, 2}(p)\right)\left\langle\psi_{k}^{-}\left(x_{2}, \cdot\right) \mid \chi_{j}\left(x_{2}, \cdot\right)\right\rangle \chi_{j}\left(x_{2}, z\right) .
\end{array}\right.
$$

The boundary conditions in $z=0$ and $z=\ell$ are due to the confinement in the transversal direction. Open boundary conditions in $x=x_{1}$ and $x=x_{2}$ comes from the fact that a wave entering the domain is partially reflected and partially transmitted between the different subbands (see [4, 25]). We define the reflection and transmission coefficients of the $k$ th subband to the $k^{\prime}$ th subband by:

$$
\begin{gathered}
\left\{\begin{array}{lr}
R_{k \rightarrow k^{\prime}}^{1}=\frac{p_{1}^{k^{\prime}}\left(E_{k, 1}(p)\right)}{p}\left|\delta_{k k^{\prime}}-\left\langle\chi_{k^{\prime}}\left(x_{1}, \cdot\right) \mid \psi_{k}^{+}\left(x_{1}, \cdot\right)\right\rangle\right|^{2} & \text { if } k^{\prime} \leq M^{1}\left(E_{k, 1}(p)\right), \\
R_{k \rightarrow k^{\prime}}^{1}=0 & \text { if } k^{\prime}>M^{1}\left(E_{k, 1}(p)\right),
\end{array}\right. \\
\begin{cases}T_{k \rightarrow k^{\prime}}^{1}=\frac{p_{2}^{k^{\prime}}\left(E_{k, 1}(p)\right)}{p}\left|\left\langle\chi_{k^{\prime}}\left(x_{2}, \cdot\right) \mid \psi_{k}^{+}\left(x_{2}, \cdot\right)\right\rangle\right|^{2} & \text { if } k^{\prime} \leq M^{2}\left(E_{k, 1}(p)\right), \\
T_{k \rightarrow k^{\prime}}^{1}=0 & \text { if } k^{\prime}>M^{2}\left(E_{k, 1}(p)\right),\end{cases}
\end{gathered}
$$

and respectively

$$
\begin{gathered}
\left\{\begin{array}{lr}
R_{k \rightarrow k^{\prime}}^{2}=\frac{p_{2}^{k^{\prime}}\left(E_{k, 2}(p)\right)}{p}\left|\delta_{k k^{\prime}}-\left\langle\chi_{k^{\prime}}\left(x_{2}, \cdot\right) \mid \psi_{k}^{-}\left(x_{2}, \cdot\right)\right\rangle\right|^{2} & \text { if } k^{\prime} \leq M^{2}\left(E_{k, 2}(p)\right), \\
R_{k \rightarrow k^{\prime}}^{2}=0 & \text { if } k^{\prime}>M^{2}\left(E_{k, 2}(p)\right),
\end{array}\right. \\
\begin{cases}T_{k \rightarrow k^{\prime}}^{2}=\frac{p_{1}^{k^{\prime}}\left(E_{k, 2}(p)\right)}{p}\left|\left\langle\chi_{k^{\prime}}\left(x_{1}, \cdot\right) \mid \psi_{k}^{-}\left(x_{1}, \cdot\right)\right\rangle\right|^{2} & \text { if } k^{\prime} \leq M^{1}\left(E_{k, 2}(p)\right), \\
T_{k \rightarrow k^{\prime}}^{2}=0 & \text { if } k^{\prime}>M^{1}\left(E_{k, 2}(p)\right) .\end{cases}
\end{gathered}
$$

We verify easily the following reciprocity relations :

$$
\begin{gathered}
R_{k \rightarrow k^{\prime}}^{1}=R_{k^{\prime} \rightarrow k}^{1}, \quad R_{k \rightarrow k^{\prime}}^{2}=R_{k^{\prime} \rightarrow k}^{2}, \\
T_{k^{\prime} \rightarrow k}^{2}\left(-p_{2}^{k^{\prime}}\left(E_{k, 1}(p)\right)\right)=T_{k \rightarrow k^{\prime}}^{1}(p), \quad T_{k^{\prime} \rightarrow k}^{1}\left(p_{1}^{k^{\prime}}\left(E_{k, 2}(p)\right)\right)=T_{k \rightarrow k^{\prime}}^{2}(-p) .
\end{gathered}
$$

Denoting

$$
\begin{aligned}
& R_{k}^{1}=\sum_{k^{\prime}} R_{k \rightarrow k^{\prime}}^{1} \quad ; \quad R_{k}^{2}=\sum_{k^{\prime}} R_{k \rightarrow k^{\prime}}^{2}, \\
& T_{k}^{1}=\sum_{k^{\prime}} T_{k \rightarrow k^{\prime}}^{1} \quad ; \quad T_{k}^{2}=\sum_{k^{\prime}} T_{k \rightarrow k^{\prime}}^{2},
\end{aligned}
$$


we obtain moreover with (2.7)-(2.8) (see [22])

$$
\begin{array}{ll}
R_{k}^{1}+T_{k}^{1}=1 & \text { for } 1 \leq k \leq M^{1}\left(E_{k, 1}(p)\right), \\
R_{k}^{2}+T_{k}^{2}=1 & \text { for } 1 \leq k \leq M^{2}\left(E_{k, 2}(p)\right) .
\end{array}
$$

A schematic representation in the phase space of the transmission and reflexion coefficients is provided in Figure 2.2. A wave entering in the quantum region $\Sigma=\left(x_{1}, x_{2}\right)$ from $x=x_{1}$ with energy $E_{k, 1}(p)$ is partially reflected with coefficient $R_{k}^{1}$ and partially transmitted with coefficient $T_{k}^{1}$.

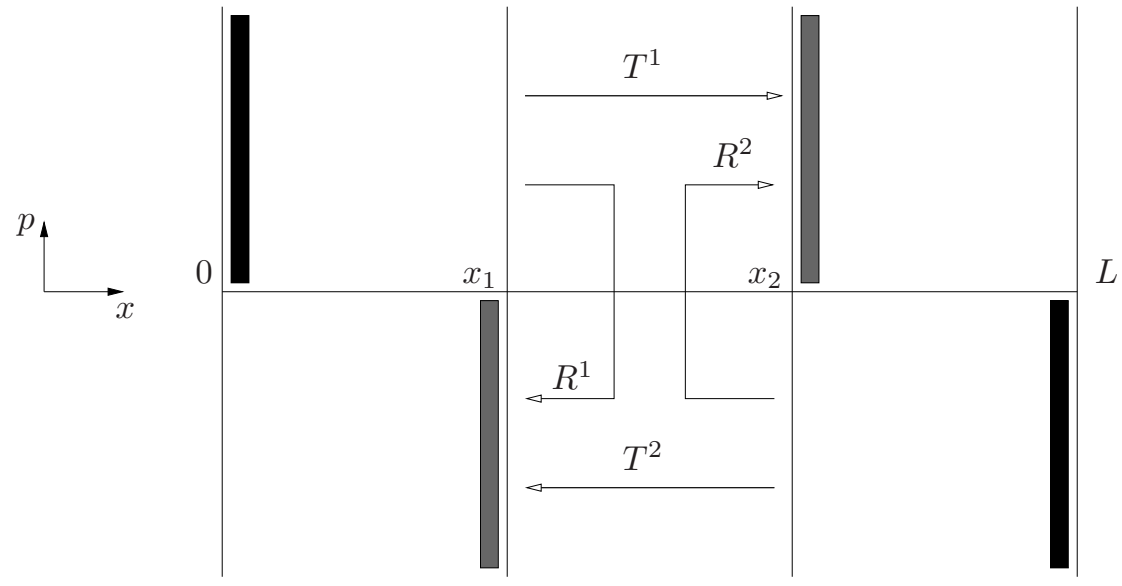

Figure 2: Schematic representation of the transmission of reflexion coefficients in phase space $(x, p)$.

We define now the two important macroscopic quantities in the quantum region: the density of electrons $N$ which enters in the right hand side of the Poisson equation (2.2) and the current. We assume temporarily that the distribution function of the electrons entering in the quantum region $\left(f_{k}(x, p)\right)_{k \geq 1}$ is known : these values being $\left(f_{k}\left(x_{1}, p\right)\right)_{k \geq 1}$ for $p>0$ and $\left(f_{k}\left(x_{2}, p\right)\right)_{k \geq 1}$ for $p<0$. The density of electrons in the quantum region is given by the sum of the contribution on each subband, from [3] (see also [22, 25]), its expression reads:

$$
N(x, z)=\frac{1}{\hbar} \sum_{k=1}^{+\infty} \int_{p>0} f_{k}\left(x_{1}, p\right)\left|\psi_{k}^{+}(x, z)\right|^{2} d p+\frac{1}{\hbar} \sum_{k=1}^{+\infty} \int_{p<0} f_{k}\left(x_{2}, p\right)\left|\psi_{k}^{-}(x, z)\right|^{2} d p .
$$

The particle current is given by:

$$
\begin{aligned}
J_{Q}(x)= & \frac{e}{m^{*}} \sum_{k=1}^{+\infty} \int_{0}^{1} \int_{p>0} f_{k}\left(x_{1}, p\right) \operatorname{Im}\left(\bar{\psi}_{k}^{+}(x, z) \partial_{x} \psi_{k}^{+}(x, z)\right) d p d z \\
& +\frac{e}{m^{*}} \sum_{k=1}^{+\infty} \int_{0}^{1} \int_{p<0} f_{k}\left(x_{2}, p\right) \operatorname{Im}\left(\bar{\psi}_{k}^{-}(x, z) \partial_{x} \psi_{k}^{-}(x, z)\right) d p d z .
\end{aligned}
$$

\section{Application of the subband decomposition method.}

Using the subband decomposition method allows to reduce the dimensionality (and consequently the cost of the numerical resolution) of the problems (2.5)-(2.6). In fact, this method consists in expanding the waves functions $\psi_{k}^{ \pm}$in the $L^{2}(0,1)$ orthonormal basis defined by the eigenfunctions $\left(\chi_{i}\right)_{i \geq 1}$ :

$$
\psi_{k}^{ \pm}(x, z)=\sum_{i=1}^{+\infty} \phi_{k i}^{ \pm}(x) \chi_{i}(x, z) .
$$


Plugging this expression into the systems of equations (2.5)-(2.6), the two dimensional stationary Schrödinger equation reduces to a one dimensional problem for the longitudinal waves $\phi_{k i}^{ \pm}$. The system reads

$$
\begin{aligned}
& -\frac{d^{2}}{d x^{2}} \phi_{k i}^{+}(x)-2 \sum_{j=1}^{\infty} a_{i j}(x) \frac{d}{d x} \phi_{k i}^{+}(x)-\sum_{j=1}^{\infty}\left(b_{i j}+\frac{2}{\hbar^{2}} c_{i j}\left(E_{k, 1}(p)-\epsilon_{j}\right)\right) \phi_{k i}^{+}(x)=0, \\
& -\frac{d^{2}}{d x^{2}} \phi_{k i}^{-}(x)-2 \sum_{j=1}^{\infty} a_{i j}(x) \frac{d}{d x} \phi_{k i}^{-}(x)-\sum_{j=1}^{\infty}\left(b_{i j}+\frac{2}{\hbar^{2}} c_{i j}\left(E_{k, 2}(p)-\epsilon_{j}\right)\right) \phi_{k i}^{-}(x)=0 .
\end{aligned}
$$

In this system the coupling terms between different subbands are given by

$$
\begin{aligned}
a_{i j} & =\int_{0}^{\ell} \chi_{i}(x, z) \frac{\partial}{\partial x} \chi_{j}(x, z) d z, \quad b_{i j}=\int_{0}^{\ell} \chi_{i}(x, z) \frac{\partial^{2}}{\partial x^{2}} \chi_{j}(x, z) d z, \\
c_{i j} & =\int_{0}^{\ell} m^{*} \chi_{i}(x, z) \chi_{j}(x, z) d z .
\end{aligned}
$$

This system is complemented with open boundary conditions. We refer interested readers to the articles [25, 22] for more details.

\subsection{Classical region}

In the classical region, the transport is mainly driven by collisions. Then we use a fluid approximation. The transport is described by the stationary drift-diffusion equation for the subband on the domain $\Omega=\left[0, x_{1}\right] \cup\left[x_{2}, L\right]$. It is a conservation law in which the current is the sum of a drift and a diffusion current. It reads [21]:

$$
\begin{aligned}
-\frac{d}{d x} J(x) & =0, \quad \text { for } x \in \Omega, \\
J(x) & =\mathbb{D}\left(\frac{d}{d x} N_{s}(x)+\beta N_{s}(x) \frac{d}{d x} U_{s}(x)\right),
\end{aligned}
$$

where $N_{s}$ is the surface density, $\mathbb{D}$ is the diffusion coefficient. This coefficient is given by the generalized Einstein rule $\mathbb{D}=\mu k_{B} T$ where $\mu$ is the electron mobility. The effective energy $U_{s}$ takes into account the confinement in the transversal direction; its expression has been derived in [7, 29]. and reads

$$
U_{s}=-k_{B} T \log \left(\sum_{k=1}^{+\infty} e^{-\beta \epsilon_{k}}\right) .
$$

In this expression the physical constants are $k_{B}$ the Boltzmann constant, $T$ the lattice temperature and $\beta=1 /\left(k_{B} T\right)$. The repartition function $\mathcal{Z}$ is defined by

$$
\mathcal{Z}(x)=\sum_{k=1}^{+\infty} e^{-\beta \epsilon_{k}(x)} .
$$

It is well-known that the drift-diffusion system can be obtained by performing a diffusive limit on the Boltzmann transport equation for semi-conductors assuming that the mean free path goes to 0 [26]. In this spirit, it has been proved in [29] that the above drift-diffusion system derives from a Boltzmann transport equation for the subband. This latter system has been used in [5, 30, to perform numerical simulations in nanoscale MOSFETs. We notice that by introducing the slotboom 
variable $u(x)=N_{s}(x) / \mathcal{Z}(x)$, the Fermi level is defined by $\epsilon_{F}(x)=k_{B} T \log \left(\frac{u(x)}{n_{i}^{2 d}}\right)$ where $n_{i}^{2 d}$ is the two dimensional intrinsic density and the current (2.14) can be written:

$$
J(x)=\mathbb{D}\left(\mathcal{Z}(x) \frac{d}{d x} u(x)\right) .
$$

To complement this system we need to define boundary conditions. At the position $x=0$ and $x=L$, due to the high doping of these regions, the drain and the source contacts can be considered as small electron reservoirs in which the surface density is constant in the vicinity of $x=0$ and $x=L$. Then we impose the Dirichlet boundary conditions:

$$
N_{s}(0)=n_{D} ; \quad N_{s}(L)=n_{D}
$$

where $n_{D}$ is the given doping profile at the source and drain contact. The difficulty consists in connecting the two open sets $\left(0, x_{1}\right)$ and $\left(x_{2}, L\right)$ thanks to interface connections. We will show in $\$ 2.4$ that imposing the continuity of the current in the device leads to the following interface conditions:

$$
\begin{gathered}
J\left(x_{1}\right)=J\left(x_{2}\right):=J \\
u\left(x_{1}\right)-u\left(x_{2}\right)=n_{i}^{2 d} e^{\beta \epsilon_{F}\left(x_{1}\right)}-n_{i}^{2 d} e^{\beta \epsilon_{F}\left(x_{2}\right)}=\theta_{Q} J
\end{gathered}
$$

where the nonnegative number $\theta_{Q}$ depends on the reflexion and transmission coefficients; as it will be detailed later its expression is given by

$$
\theta_{Q}=\left(\frac{e}{m^{*} \hbar} \sum_{k=1}^{+\infty} \int_{0}^{+\infty} p T_{k}^{1}(p) M(p) e^{-\beta \epsilon_{k}\left(x_{1}\right)} d p\right)^{-1}, \quad \text { where } M(p)=\frac{1}{\pi} e^{-\beta p^{2} /\left(2 m^{*}\right)} .
$$

Finally, the stationary problem defining the classical region is given by the system (2.13)-(2.14) coupled with boundary conditions (2.18) $-(2.19)-(2.20)$.

Knowing the surface density $N_{s}$ and assuming to be at thermal equilibrium, the distribution function in the classical region is given by the Maxwellian:

$$
f_{k}(x, p)=N_{s}(x) \mathcal{M}_{k}(p)=u(x) \mathcal{Z}(x) \mathcal{M}_{k}(p) ; \quad \mathcal{M}_{k}(p)=\frac{1}{\pi \mathcal{Z}} \exp \left(-\frac{\beta p^{2}}{2 m^{*}}-\beta \epsilon_{k}(x)\right) .
$$

The Maxwellian $\mathcal{M}$ is normalized such that $\frac{1}{\hbar} \sum_{k} \int_{\mathbb{R}} \mathcal{M}_{k}(p) d p=n_{i}^{1 d}$ where $n_{i}^{1 d}=2 \sqrt{\frac{m^{*}}{2 \pi \beta \hbar^{2}}}$ is the one dimensional intrinsic density. The occupation number of the $k$ th subband is given by

$$
\rho_{k}(x)=\frac{1}{\hbar} \int_{\mathbb{R}} f_{k}(x, p) d p=n_{i}^{1 d} u(x) e^{-\beta \epsilon_{k}(x)} .
$$

Finally the total density of electrons in the classical region is given by

$$
N(x, z)=\sum_{k=1}^{+\infty} \rho_{k}(x)\left|\chi_{k}(x, z)\right|^{2}=n_{i}^{1 d} \frac{N_{s}(x)}{\mathcal{Z}(x)} \sum_{k=1}^{+\infty} e^{-\beta \epsilon_{k}(x)}\left|\chi_{k}(x, z)\right|^{2} .
$$

This quantity will be injected in the right hand side of the Poisson equation (2.2) in the classical region. 


\subsection{Interface conditions}

In this Section, we derive the interface conditions (2.19)-(2.20) and give an expression of the constant $\theta_{Q}$ in these boundary conditions. In the previous work of Baro et al. [1], it has been observed that interface conditions can be obtained by imposing the continuity of the current inside the device. In fact, this property is important in such electronic devices [3, 1].

Proposition 2.1 Let us consider the domain $(0, L)=\Omega \cup \Sigma$, where the current is defined for $x \in \Sigma$ by (2.12) and for $x \in \Omega$ by (2.14). In these definitions $\psi_{k}^{ \pm}$solve (2.5)-(2.6) and $N_{s}$ is a solution of the drift-diffusion system (2.13). Then, if the connection conditions (2.19) $-(2.20)$ at interfaces $x=x_{1}$ and $x=x_{2}$ are satisfied with $\theta_{Q}$ given by (2.21), the current is continuous and constant in $(0, L)$.

\section{Proof.}

The current is constant. First, the stationary drift-diffusion system (2.13) verifies straightforwardly that the current is constant in the classical regions. Then, using expression (2.12) of $J_{Q}$ we can show that the current in the quantum region is also constant. In fact, using the Schrödinger equations for the wave functions $\psi_{k}^{+}(2.5)$ and $\psi_{k}^{-}$(2.6), we have

$$
\partial_{x} \operatorname{Im}\left(\bar{\psi}_{k}^{ \pm}(x, z) \partial_{x} \psi_{k}^{ \pm}(x, z)\right)=\operatorname{Im}\left(\bar{\psi}_{k}^{ \pm}(x, z) \partial_{x x} \psi_{k}^{ \pm}(x, z)\right)=-\operatorname{Im}\left(\bar{\psi}_{k}^{ \pm}(x, z) \partial_{z z} \psi_{k}^{ \pm}(x, z)\right) .
$$

Then, integrating for $z \in(0, \ell)$, using an integration by parts and the confining boundary conditions $\psi_{k}^{ \pm}(x, 0)=\psi_{k}^{ \pm}(x, \ell)=0$, we deduce

$$
\int_{0}^{\ell} \partial_{x} \operatorname{Im}\left(\bar{\psi}_{k}^{ \pm}(x, z) \partial_{x} \psi_{k}^{ \pm}(x, z)\right) d z=0,
$$

since the imaginary part only holds on real numbers. Consequently, the current is constant in each region of the device. Therefore it is continuous in the whole device providing the constant in each region is the same.

Continuity in the classical region. Equation (2.19) implies that the constant value of the classical current is the same in each classical region.

Continuity with quantum current. Then it is enough now to impose the equality of the constant quantum current with the constant classical current. To do so, we first observe that with the open boundary conditions in (2.5) $-(2.6)$, we have

$$
\int_{0}^{\ell} \operatorname{Im}\left(\bar{\psi}_{k}^{ \pm}\left(x_{1}, z\right) \partial_{x} \psi_{k}^{ \pm}\left(x_{1}, z\right)\right) d z=\int_{0}^{\ell} \operatorname{Im}\left(\bar{\psi}_{k}^{ \pm}\left(x_{2}, z\right) \partial_{x} \psi_{k}^{ \pm}\left(x_{2}, z\right)\right) d z .
$$

This latter equality and the definition of the reflexion and transmission coefficients allow to obtain after integrating the quantum current $J_{Q}$ in (2.12) on $\left[x_{1}, x_{2}\right]$ :

$$
J_{Q}=\frac{e}{m^{*} \hbar} \sum_{k=1}^{+\infty} \int_{0}^{+\infty}\left(p T_{k}^{1}(p) f_{k}\left(x_{1}, p\right)-p T_{k}^{2}(p) f_{k}\left(x_{2},-p\right)\right) d p .
$$

We recall the expression of the distribution function (2.22) rewritten using the Slotboom variable $u=N_{s} / \mathcal{Z}$ :

$$
f_{k}(x, p)=u(x) M(p) e^{-\beta \epsilon_{k}(x)} ; \quad \text { where } M(p)=\frac{1}{\pi} e^{-\beta p^{2} /\left(2 m^{*}\right)} .
$$


Injecting this latter expression in the expression of the quantum current given in (2.25), we get after a change of variable and using the reciprocity relations (2.8) and (2.9),

$$
J_{Q}=\left(u\left(x_{1}\right)-u\left(x_{2}\right)\right) \frac{e}{m^{*} \hbar} \sum_{k=1}^{+\infty} \int_{0}^{+\infty} p T_{k}^{1}(p) M(p) e^{-\beta \epsilon_{k}\left(x_{1}\right)} d p .
$$

Since we want that the constant $J_{Q}$ coincides with the constant $J$, from the definition of $\theta_{Q}$ in (2.21), condition (2.26) rewrites as condition (2.20). It concludes the proof.

\section{Numerical results}

\subsection{Algorithm}

Let us introduce a discretization of the domain in the $x$ direction $[0, L]$ with a cartesian grid whose nodes are denoted $x_{i}, i=1, \ldots, N_{x}$, and a discretization of the domain in the $z$ direction $[0, \ell]$ by a cartesian grid with nodes $z_{j}, j=1, \ldots, N_{z}$. The classical region is discretized with $2 \times N_{c}$ nodes, for $x_{i}=1, \ldots, N_{c}$ and $x_{i}=N_{x}-N_{c}+1, \ldots, N_{x}$. Then, we mesh the domain $[0, L] \times[0, \ell]$ with rectangular triangles using the nodes $\left(x_{i}, z_{j}\right)$ defined above. The one-dimensional Schrödinger equations in the transversal direction $z$ and the Poisson equation are discretized thanks to $P^{1}$ finite elements. In the classical region, the one-dimensional drift-diffusion system is discretized thanks to the Scharfetter-Gummel scheme as in [27, 11, 23]. In the quantum region, Schrödinger equations in the transport direction (2.5) -(2.6) are treated with the WKB method presented in [8].

The algorithm consists in two main steps: firstly we compute the equilibrium when there is no applied drain-source bias, secondly we consider the resolution of the whole system by incrementing small drain-source bias from the equilibrium quantities.

At equilibrium, with no applied drain-source bias, the Fermi-level (or equivalently the slotboom variable) is constant and is then determined by its value at the frontier. Therefore there is no need to solve the equations in the transport direction in this case. Thus the problem boils down to the resolution of the diagonalization of the 1D Schrödinger operator (2.1) coupled to the 2D Poisson equation (2.2) -(2.3) where the density $N$ is given in the classical region by (2.23), where $u$ is constant, and $N$ is given in the quantum region by (2.11), where the distribution function $f_{k}$ in this expression is at equilibrium and given by (2.22).

Once the equilibrium potential is computed, we increment the drain-source voltage by steps of $0.02 \mathrm{~V}$. Then the Fermi level is not constant and we need to solve the whole system. To do so, we consider the following Gummel iterative process:

1. For a given potential $V_{\text {old }}$ (which initially is taken to be the computed equilibrium potential), we solve the eigenvalues problems (2.1) where $U=-e V_{\text {old }}$ by diagonalization of the Hamiltonian on each slice of the device $\left(x=x_{i}, i=1, \ldots, N_{x}\right)$. We obtain $N_{x}$ sets of eigenvalues $\left(\epsilon_{k}\left(x_{i}\right)\right)_{k \geq 1}$ and eigenfunctions $\left(\chi_{k}\left(x_{i}, z\right)\right)_{k \geq 1}$ for $i=1, \ldots, N_{x}$.

2. We solve the problem in the quantum region by resolution of the problems (2.5)-(2.6). To do so we use the SDM/WKB method which has been described in 88. Then we have everything at hand to compute the reflexion and transmission coefficients in the quantum region defined in 92.2 . A value of the coupling constant $\theta_{Q}$ is obtained from (2.21). 
3. Knowing $\theta_{Q}$, we can solve the $1 \mathrm{D}$ drift-diffusion system (2.13) with boundary conditions (2.18) (2.20) in the classical region. The Scharfettel-Gummel scheme is used for this resolution [27, 11, 23].

4. An alimentation function $f_{k}$ can be computed with expression (2.22). Then, we compute the total density of electrons thanks to (2.11) in the quantum region, and (2.23) in the classical region.

5. The Poisson equation (2.2) $-(2.3)$ is solved in the $2 \mathrm{D}$ computational domain. A new value of the potential $V_{\text {new }}$ is obtained. In our algorithm, the Poisson equation is solved using the preconditioned conjugate gradient method.

6. We repeat the last five steps with this new value of the potential until the error $\left\|V_{\text {new }}-V_{\text {old }}\right\|_{L^{\infty}}$ is small enough.

\subsection{Numerical simulations of a Double-Gate MOSFET}

To illustrate our algorithm, we simulate the transport of charged carriers in a nanotransistor DoubleGate MOSFET. In such a device, the active region where transport occurs is insulated from ohmic contacts of the gates thanks to oxide layers. A strong barrier potential $U_{c}$ at the oxide-silicon interface implies a confinement in the direction $z$. Moreover, due to doping of the source and drain region, collisions are important in these regions of the device, whereas in the channel between these two regions, tunneling effects occur. Then the model presented above is relevant to simulate the transport of charged carriers in this device.

The computational domain is $(x, z) \in[0, L] \times[0, \ell]$. The classical region is the union of the source zone $\left[0, x_{1}\right] \times[0, \ell]$ and of the drain zone $\left[x_{2}, L\right] \times[0, \ell]$, with $0<x_{1}<x_{2}<L$. The length of the source is then $L_{S}=x_{1}$; the length of the channel is denoted $L_{C}=x_{2}-x_{1}$; therefore the length of the drain is $L_{D}=L-x_{2}$ (see Figure 3.2). We denote $\ell_{\text {Grid }}$ the length of the Gates. We will set $\Sigma=\left(x_{1}, x_{2}\right)$ and $\Omega=(0, L) \backslash \Sigma$. A Gate potential $V_{G}$ can be applied at the gate and a drain-source $V_{D S}$ potential can be applied between the ohmic contacts of the drain and of the source. These ohmic contacts define the part of the boundary $\Gamma_{D}$ where Dirichlet boundary conditions are used in the Poisson equation (2.3) $: \Gamma_{D}=(\{0\} \cup\{L\}) \times[0, \ell] \cup\left[\frac{L-\ell_{\text {Grid }}}{2}, \frac{L+\ell_{\text {Grid }}}{2}\right] \times(\{0\} \cup\{\ell\})$. The remaining boundary is $\Gamma_{N}$ where Neumann boundary conditions are used.

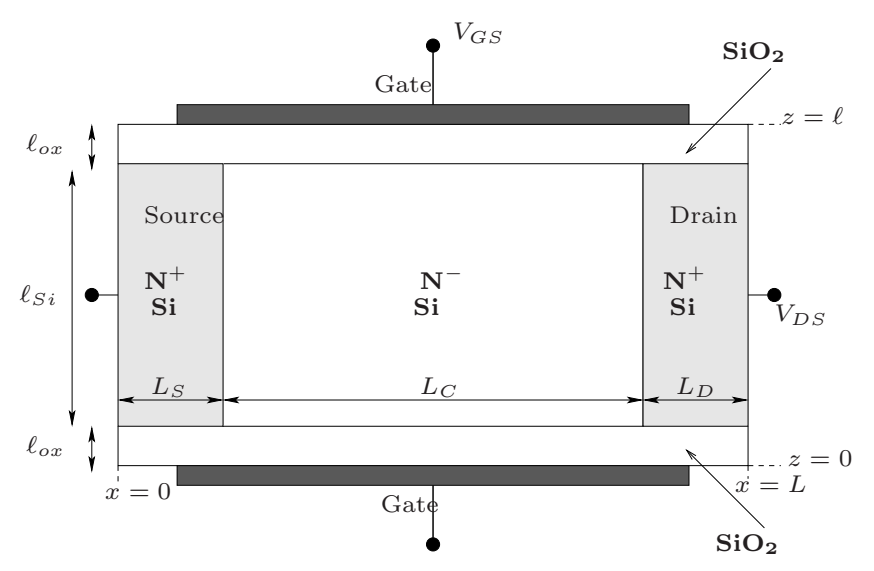

Figure 3: Schematic representation of a Double-Gate MOSFET. 
For the numerical simulations, we use the physical values gathered in table 1 . Moreover, we take $N_{x}=91$ nodes, $N_{c}=21$ nodes and $N_{z}=36$ nodes. Finally, only a few number of eigenenergies $\epsilon_{k}$ are used. In fact, the expression of the distribution function in (2.22) depends exponentially of eigenenergies. The numerical results presented here have been obtained with 6 modes $\epsilon_{k}, k=$ $1, \ldots, 6$.

Table 1: Table of the main physical values

\begin{tabular}{|c||c||c||c|}
\hline Parameter & Value & Length & Value \\
\hline \hline$N^{+}$ & $10^{20} \mathrm{~cm}^{-3}$ & $L_{S}$ & $4 \mathrm{~nm}$ \\
\hline$N^{-}$ & $10^{15} \mathrm{~cm}^{-3}$ & $L_{C}$ & $10 \mathrm{~nm}$ \\
\hline$U_{c}$ & $3 \mathrm{eV}$ & $L_{D}$ & $4 \mathrm{~nm}$ \\
\hline$\varepsilon_{R}[\mathrm{Si}]$ & 11.7 & $\ell_{\text {ox }}$ & $1 \mathrm{~nm}$ \\
\hline$\varepsilon_{R}\left[\mathrm{SiO} \mathrm{O}_{2}\right]$ & 3.9 & $\ell_{S i}$ & $5 \mathrm{~nm}$ \\
\hline$\mu$ & $0.12 \mathrm{~m}^{2} V^{-1} \mathrm{~s}^{-1}$ & $\ell_{\text {grid }}$ & $12 \mathrm{~nm}$ \\
\hline \multicolumn{3}{|l}{} \\
\hline
\end{tabular}

\subsubsection{Results obtained with the hybrid approach}

Figure 4 shows the electron density for a drain-source voltage $V_{D S}=0 \mathrm{~V}$ (left) and $V_{D S}=0.4 \mathrm{~V}$ (right). In these simulations there is no applied Gate bias $\left(V_{G}=0 V\right)$. Without applied voltage $\left(V_{D S}=0 \mathrm{~V}\right)$, the system is at equilibrium and we observe that the electron density is symmetric. Applying a bias, the density does not remain symmetric. The electrons are drifted in the direction of lower potential energy and accumulate in the drain region. It points out the transport of electrons in the device. We make the same observation in Figure 5, where we display the potential energy $U$ when there is no applied drain-source voltage (left) and for $V_{D S}=0.4 V$ (right).
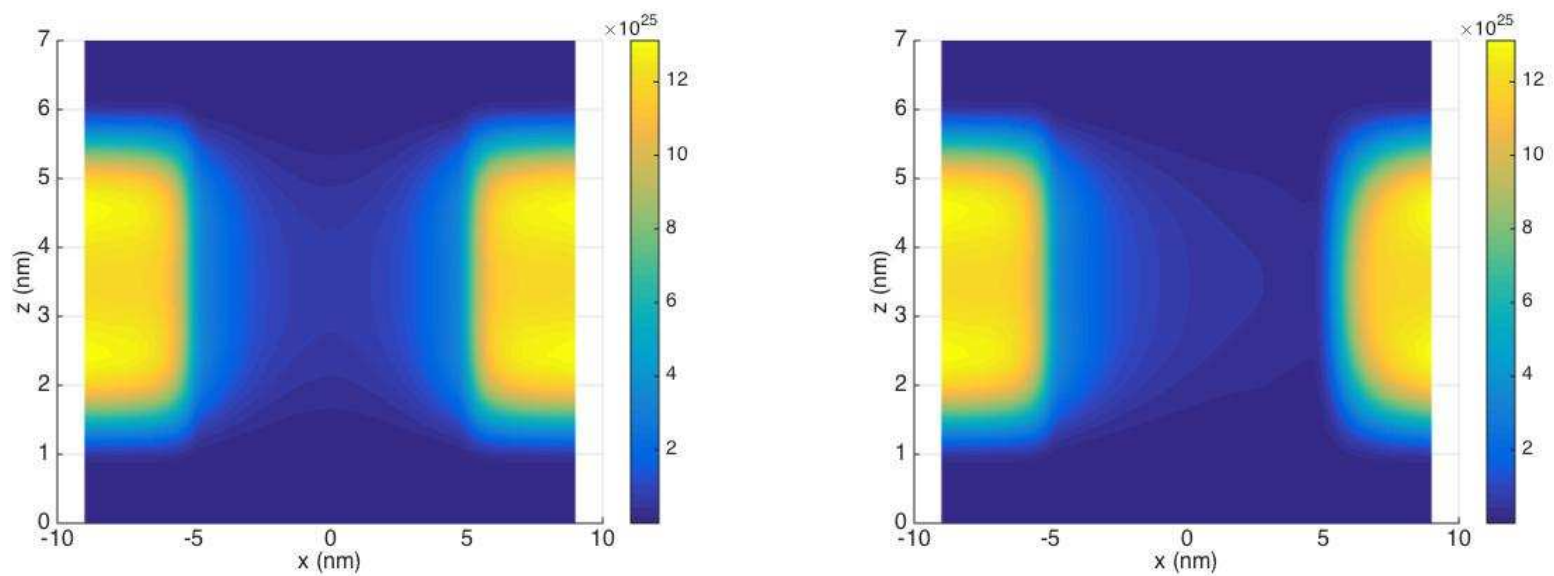

Figure 4: Electron density $\left(\mathrm{m}^{-3}\right)$ for $V_{G}=0 \mathrm{~V}$ at equilibrium (left) and for $V_{D S}=0.4 \mathrm{~V}$ (right).

In Fig 6, 1D profiles of the surface electron density (left) and of the potential energy (right) are presented for $V_{D S}=0.4 \mathrm{~V}$ with different gate voltages. These $1 \mathrm{D}$ curves are averaged quantities resulting from an integration of the $2 \mathrm{D}$ quantities over the transversal section. For $V_{G}=-0.1 \mathrm{~V}$, the transport is mainly controlled by the gate: the potential inside the channel has the same value than 

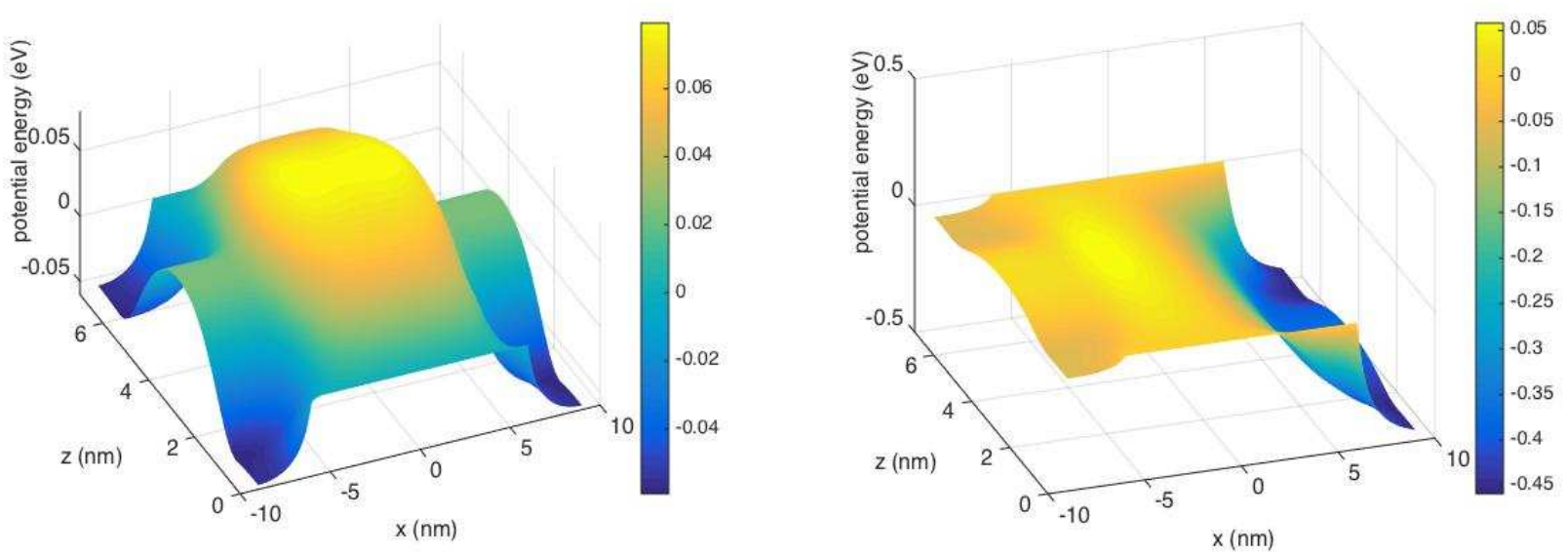

Figure 5: Potential energy (eV) for $V_{G}=0 \mathrm{~V}$ at equilibrium (left) and for $V_{D S}=0.4 \mathrm{~V}$ (right).

$V_{G}$. As $V_{G}$ increases, the density inside the channel and consequently the electron transport also increases. However, for large $V_{G}$, the action of the gate is less pronounced and the potential inside the channel does not reach the gate value.
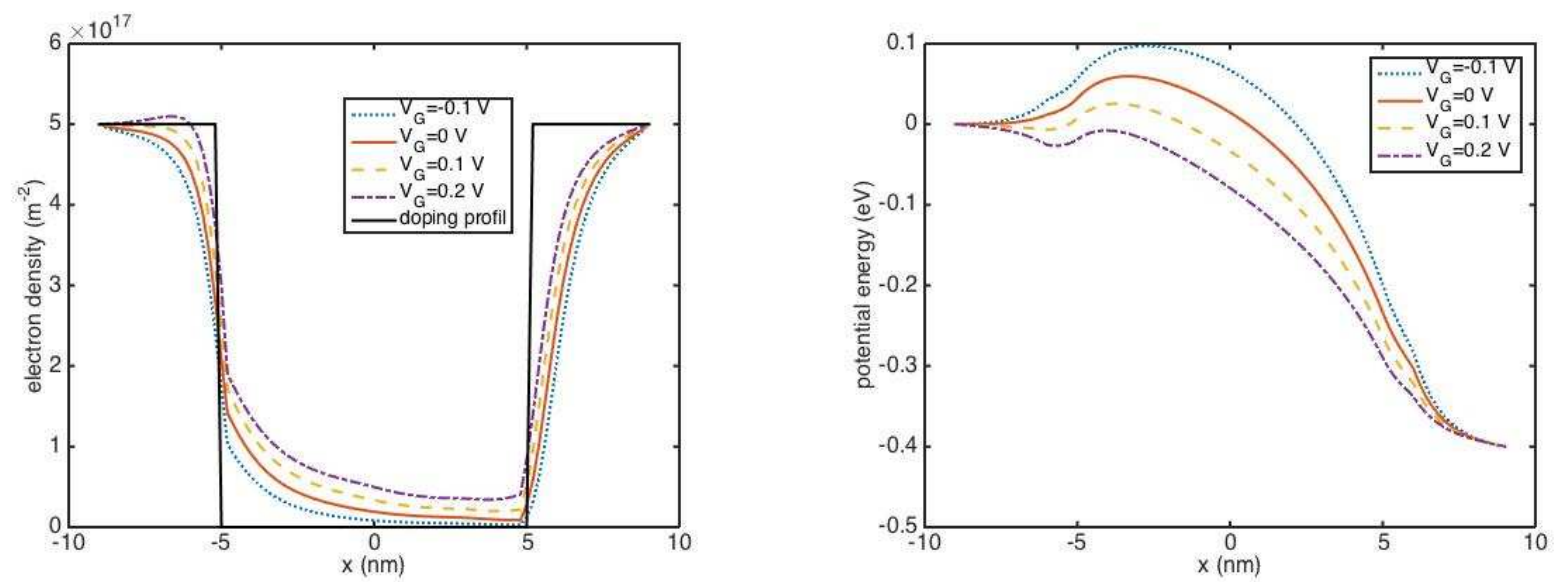

Figure 6: Surface electron density $\left(\mathrm{m}^{-2}\right)$ (left) and potential energy (eV) (right) for $V_{D S}=0.4 \mathrm{~V}$ and different gate voltages $V_{G}$.

The current-voltage characteristics are given in Figure 7, Figure 7-left displays the current vs drain-source applied bias. Figure 7-right displays in semilog scale the current vs $V_{G}$. As it was announced by Fig, 6, the current increases with the gate voltage $V_{G}$.

\subsubsection{Comparison of the three approaches}

Now, we shall compare three different approaches (classical, quantum and hybrid). In each case, the subband decomposition method is used to split the transport and the confining direction. In the transversal direction, electrons are always described by a statistical mixture of eigenstates of the Schrödinger operator. It is the strategy used to describe the electron motion in the transport direction that is changed. More precisely, the hybrid approach described in this paper (dashed 

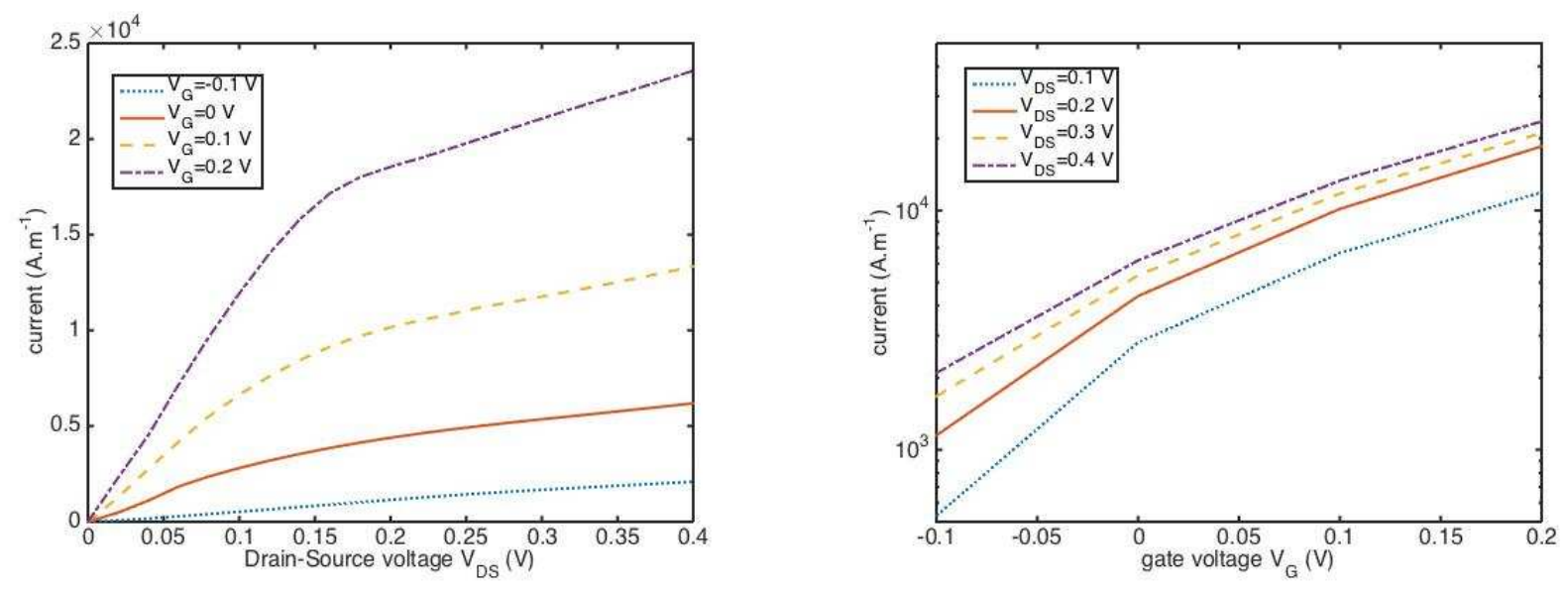

Figure 7: Current-Voltage characteristics as a function of $V_{D S}$ (left) and of $V_{G}$ (right).

yellow curves in Figs. 8, 10] is compared with the fully quantum model [25, 8] (dotted red curves) and with the quantum-diffusive approach [23] consisting in using the drift-diffusion model in the entire transport domain $[0, \mathrm{~L}]$ (dashdotted blue curves).

Since the 2D profils of the electron density and of the potential are almost similar between the three approaches, we prefer to present the 1D averaged quantities in order to emphasize the differences. In Fig 8 -left, the surface electron density is presented at equilibrium for $V_{G}=0 \mathrm{~V}$. For a better visualization, it is also interesting to look at its inverse (Fig 8-left). Clearly, we recover that the hybrid approach matches with the drift-diffusion one at reservoirs (source and drain regions). On the contrary, inside the active zone, where quantum effects are predominant, the results obtained with the coupling approach tend to reach the fully quantum ones. The same behavior is observed in Fig 9 where the inverse of the surface density (left) and the potential energy (right) are plotted for $V_{D S}=0.4 \mathrm{~V}$.
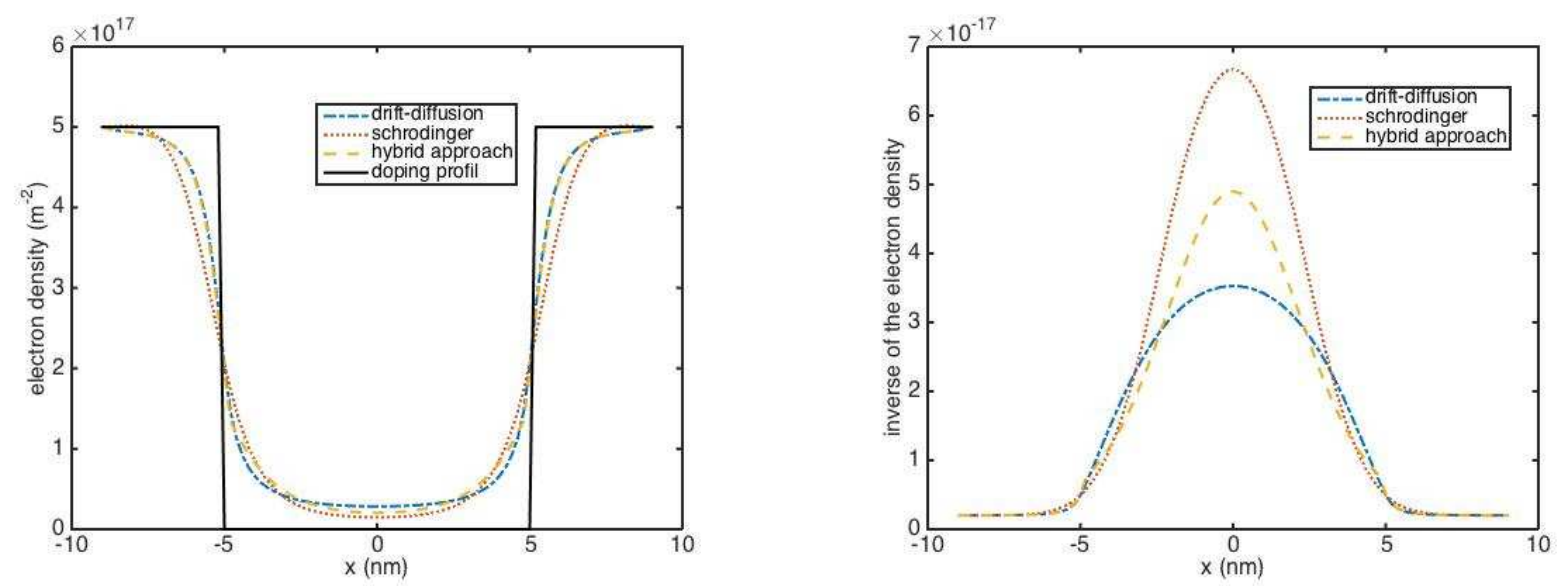

Figure 8: Surface electron density $\left(\mathrm{m}^{-2}\right)$ (left) and its inverse (right) at equilibrium for $V_{G}=0 \mathrm{~V}$.

Finally, the current-voltage characteristics are compared in Figure 10 (current vs $V_{D S}$ in the left and current vs $V_{G}$ in the right). With a coupling approach, the electron transport is strongly 

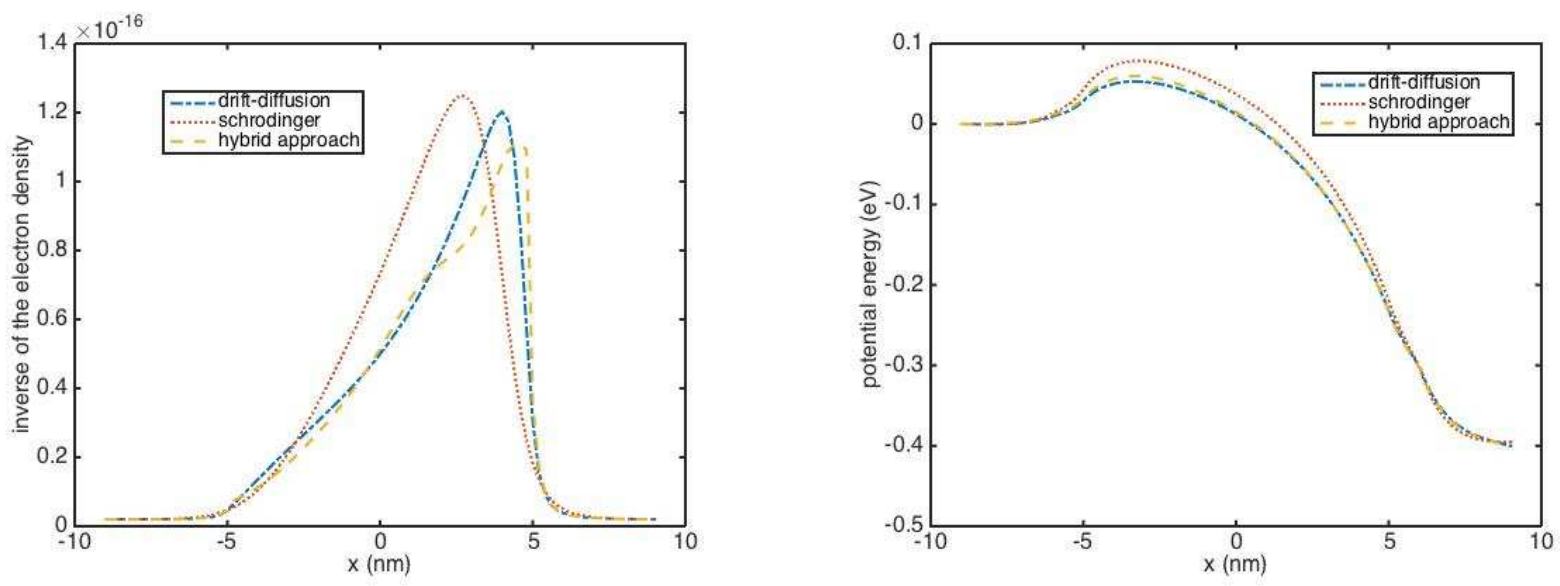

Figure 9: Inverse of the surface electron density $\left(\mathrm{m}^{2}\right)$ and potential energy $(\mathrm{eV})$ (right) for $V_{D S}=$ $0.4 \mathrm{~V}$ and $V_{G}=0 \mathrm{~V}$.

affected not only by collisions at reservoirs but also by quantum effects inside the channel. It seems relevant to observe an intermediate current value.
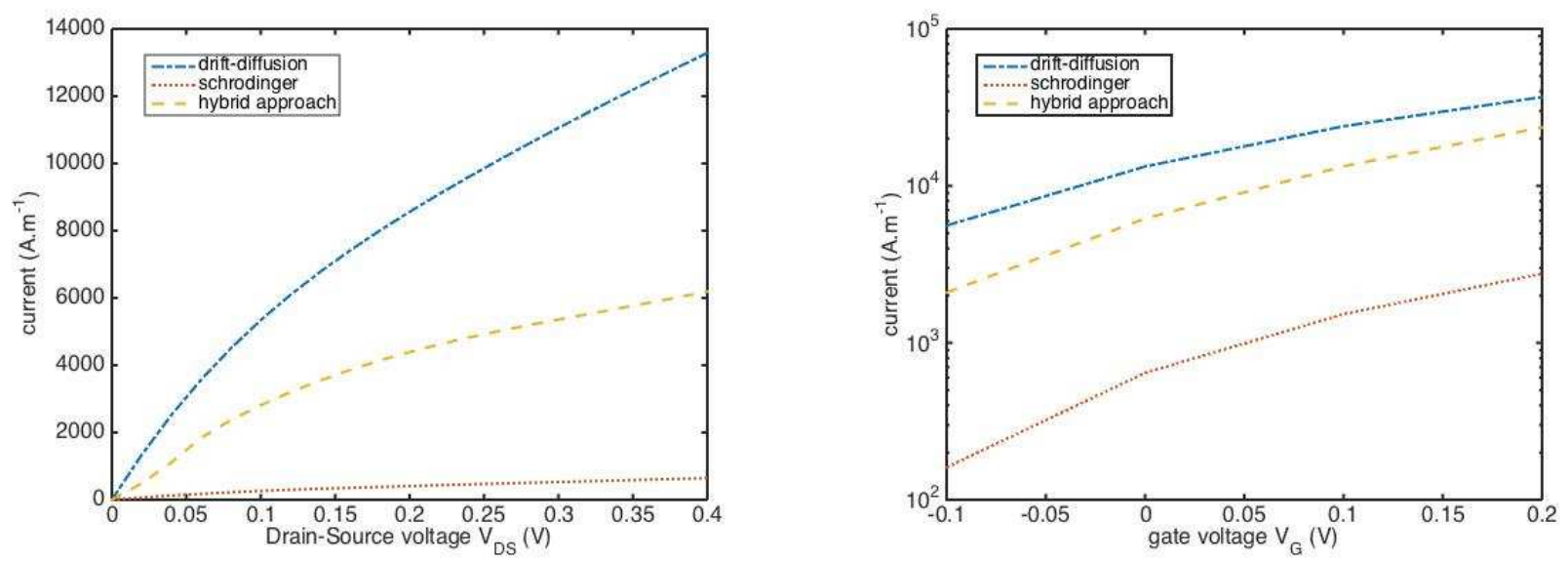

Figure 10: Comparison of the current-Voltage characteristics between the three approaches as a function of $V_{D S}$ for $V_{G}=0 \mathrm{~V}$ (left) and of $V_{G}$ for $V_{D S}=0.4 \mathrm{~V}$ (right).

\subsubsection{Interface positions}

Finally, we study the influence of the interface positions for the coupling approach. In the previous simulations, the interfaces $x_{1}$ and $x_{2}$ are located at the doping discontinuities (4 $\mathrm{nm}$ far from the boundaries). It is called "no shift" case in the sequel. In Figs 11, 12, the two interfaces are moved at the same time of 0.2 or $0.4 \mathrm{~nm}$, either inside or outside the channel. The inverse of the density is reported in Fig 11 at equilibrium (left) and for $V_{D S}=0.4 \mathrm{~V}$ (right) for $V_{G}=0 \mathrm{~V}$. We recall that the interface conditions (2.19)-(2.20) are built preserving the current continuity. Consequently, density discontinuity may occur at interfaces, as observed in Fig [1]. 

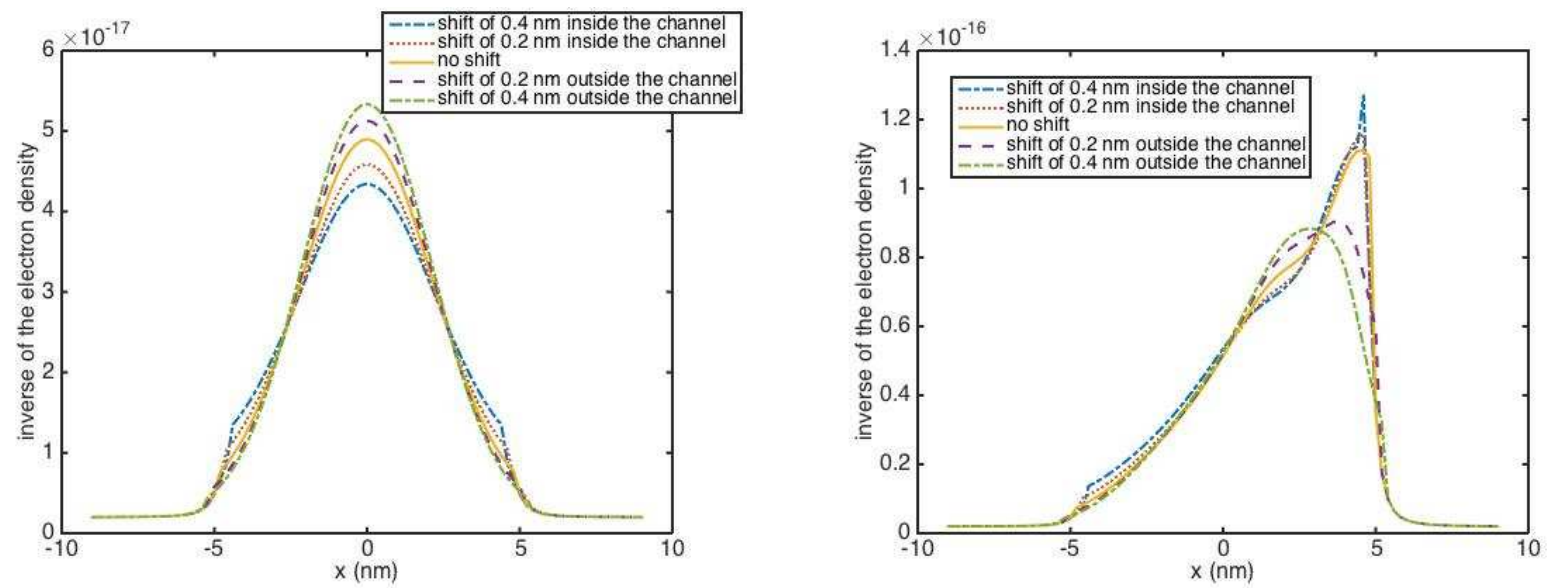

Figure 11: Inverse of the electron density $\left(\mathrm{m}^{2}\right)$ at equilibrium (left) and for $V_{D S}=0.4 \mathrm{~V}$ (right) for $V_{G}=0 \mathrm{~V}$ obtained with the hybrid approach moving the interfaces.

The current-voltage characteristics are presented in Fig 12. When the quantum zone is reduced (interfaces moved inside the channel), the current value gets closer to the one obtained with the full drift-diffusion model, and inversely. We point out that a similar dependence on the interface position occurs for different gate voltages.

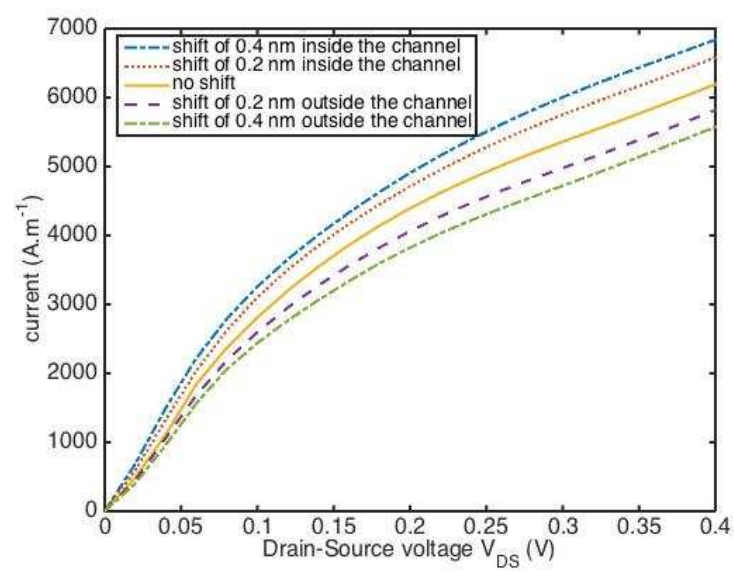

Figure 12: Current-Voltage characteristics for $V_{G}=0 \mathrm{~V}$ obtained with the hybrid approach moving the interfaces.

\section{Conclusion}

A numerical model describing the confined transport of electrons in a nano DG-MOSFET has been presented. This model couples a classical description at diffusive regime in the exterior reservoirs with a purely quantum description in the active zone. This coupling occurs in the transport direction whereas particles are confined in the transverse direction. More precisely, the drift-diffusion-Poisson system is coupled to the Schrödinger-Poisson system through interface conditions in the transport 
direction. The quantum direction is modeled thanks to the subband decomposition method. Numerical simulations for a DG-MOSFET are provided.

Several extensions of this work can be considered. First, concerning numerical simulations of the DG-MOSFET considered in this work, several models describing the transport in the collisional region in the channel have been proposed depending on the accuracy of the collision description. Actually a hierarchy of models for confined electrons has been proposed in [6 where the classical transport is modeled by the Boltzmann transport equation at the mesoscopic level of description. From this kinetic model, assuming that elastic collisions are dominant, the Boltzmann transport equation can be approximated by the spherical harmonic expansion (SHE) model. Assuming then that electron-electron scattering is dominant, the latter system can be approximated by the energytransport (ET) model. Thus an extension of this work consists in adapting our coupling strategy to this hierarchy of model. A first step consists in deriving coupling conditions for the SHE and ET model in the fully classical case. It is the topic of the recent work [18.

Furthermore, the approach used in this paper can also be applied to other devices for which an electron gas is confined in one or several directions and where the ballistic transport is located in a well localized region of the device. For instance, a similar hybrid strategy has been applied in [17. in the framework of strongly confined nanostructures (like nanowires or nanotubes) using models that take into account the peculiarities due to the two dimensional transversal crystal structure. In this work, numerical simulations of a gate-all-around Carbon Nanotube Field-Effect Transistor are presented.

Acknowledgments. The authors would like to dedicate this work to Naoufel Ben Abdallah who was a talented researcher, an enthusiastic supervisor and a generous person. The authors would like to acknowledge warmly Claudia Negulescu for her kind assistance.

\section{References}

[1] M. Baro, N. Ben Abdallah, P. Degond, A. El Ayyadi, A 1D coupled Schrödinger drift-diffusion model including collisions, J. Comp. Phys 203 (2005), 129-153.

[2] G. Bastard, Wave mechanics applied to semiconductor heterostructures, Les éditions de Physique (1996)

[3] N. Ben Abdallah, A Hybrid Kinetic-quantum model for stationary electron transport, J. Stat. Phys. 90 no 3-4 (1998), 627-662.

[4] N. Ben Abdallah, On a multidimensional Schrödinger-Poisson scattering model for the semiconductors, J. Math. Phys. 41 no 3-4 (2000), 4241-4261.

[5] N. Ben Abdallah, M. J. Cáceres, J. A. Carrillo, F. Vecil, A deterministic solver for a hybrid quantum-classical transport model in nanoMOSFETs, J. Comput. Phys. 228 (2009), no. 17, 6553-6571.

[6] N. Ben Abdallah, F. Méhats, C. Negulescu, Adiabatic quantum-fluid transport models, Commun. Math. Sci. 4 (2006), no. 3, 621-650.

[7] N. Ben Abdallah, F. Méhats, N. Vauchelet, Diffusive transport of partially quantized particles : existence uniqueness and long time behaviour, Proc. Edinb. Math. Soc. (2006) 49, 513-549. 
[8] N. Ben Abdallah, M. Mouis, C. Negulescu, An accelerated algorithm for 2D simulations of the quantum ballistic transport in nanoscale MOSFETs, J. Comput. Phys. 225 (2007), no. 1, 74-99.

[9] N. Ben Abdallah, O. Pinaud, Multiscale simulation of transport in an open quantum system: resonances and WKB interpolation, J. Comput. Phys., 213 (1) (2006), 288-310.

[10] N. Ben Abdallah, S. Tang, On hybrid quantum-classical transport models, Math. Meth. Appl. Sci. 27 (2004), 643-667.

[11] F. Brezzi, L.D. Marini, P. Pietra, Méthodes d'éléments finis mixtes et schéma de ScharfetterGummel, C.R. Acad. Sci. Paris Sér. I, 305 (1987), 599-604.

[12] J.-P. Colinge, FinFETs and Other Multi-Gate Transistors, first ed., Springer Publishing Company, Incorporated, 2007.

[13] J. H. Davies, The Physics of Low Dimensional Semiconductors, Cambridge Univ. Press, Cambridge (1998)

[14] P. Degond, A. El Ayyadi, A coupled Schrödinger Drif-Diffusion model for Quantum semiconductor device simulations, J. Comp. Phys. 181 (2002), 222-259.

[15] International technology roadmap for semiconductor industry. URL: http://www.itrs.net/.

[16] D. K. Ferry, S. M. Goodnick, Transport in Nanostructures. Cambridge Univ. Press, Cambridge (1997)

[17] C. Jourdana, P. Pietra, A hybrid classical-quantum transport model for the simulation of Carbon Nanotube transistors, SIAM J. Sci. Comput., 36 (2014), no. 3, B486-B507.

[18] C. Jourdana, P. Pietra, N. Vauchelet, A classical-quantum coupling strategy for a hierarchy of one dimensional models for semiconductors, submitted

[19] A. Jüngel, Transport equations for semiconductors. Lecture Notes in Physics, 773. SpringerVerlag, Berlin, 2009.

[20] C.S. Lent, D.J. Kirkner, The quantum transmitting boundary method, J. Appl. Phys., 67 (1990), pp. 6353-6359.

[21] P. A. Markowich, C. A. Ringhofer, C. Schmeiser, Semiconductor equations, Springer-Verlag, Vienna, 1990.

[22] C. Negulescu, Small coherence length limit for a two dimensional quantum transport model, Asymptotic Analysis 49, no. 3-4 (2006), 295-329.

[23] P. Pietra, N. Vauchelet, Modeling and simulation of the diffusive transport in a nanoscale Double-Gate MOSFET, J. Comput. Elec. (2008) 7:52-65.

[24] P. Pietra, N. Vauchelet, Numerical simulations of an energy-transport model for partially quantized particles, Commun. Math. Sci. 12 (2014), no. 1, 99-123.

[25] E. Polizzi, N. Ben Abdallah, Subband decomposition approach for the simulation of quantum electron transport in nanostructures, J. Comp. Phys. 202 (2005), 150-180.

[26] F. Poupaud, Diffusion approximation of the linear semiconductor Boltzmann equation: analysis of boundary layers, Asymptotic Analysis 4 (1991), 293-317. 
[27] D.L. Scharfetter, H.K. Gummel, Large signal analysis of a silicon Read diode oscillator. IEEE Trans. Electron Devices ED-16 (1969), 64-77.

[28] N. Vauchelet, Diffusive transport of partially quantized particles : LlogL solutions, Math. Models Methods Appl. Sci. (2008), Vol 18 no 4, 489-510.

[29] N. Vauchelet, Diffusive limit of a two dimensional kinetic system of partially quantized particles, J. Stat. Phys. (2010) 139, 882-914.

[30] F. Vecil, J. M. Mantas, M. J. Cáceres, C. Sampedro, A. Godoy, F. Gámiz, A parallel deterministic solver for the Schrödinger-Poisson-Boltzmann system in ultra-short DG-MOSFETs: comparison with Monte-Carlo, Comput. Math. Appl. 67 (2014), no. 9, 1703-1721.

[31] R. Venugopal, Z. Ren, S. Datta, M. S. Lundstrom, Simulating quantum transport in nanoscale transistor : Real versus mode-space approaches, J. Appl. Phys 92 (2002), n 7, 3730-3729. 